\title{
Impaired CD4-CELl ImMune ReCONSTITUtion UPON HIV Therapy in Patients with Toxoplasmic Encephalitis Compared to Patients with Pneumocystis Pneumonia as AIDS Indicating Disease
}

\author{
U. Kastenbauer ${ }^{1}$, E. Wolf ${ }^{2}$, C. Kollan ${ }^{3}$, O. Hamouda ${ }^{3}$, J. R. Bogner ${ }^{1}$ for the ClinSurv Study Group \\ ${ }^{1}$ Department of Infectious Diseases, University Hospital Munich, Downtown Campus, Medizinische Poliklinik, Munich, Germany, \\ ${ }^{2}$ MUC Research, Munich, Germany, ${ }^{3}$ Robert Koch-Institut, Berlin, Germany
}

\begin{abstract}
Objectives: There is only little data on immune reconstitution in antiretroviral naïve AIDS-patients with toxoplasmosis. The observation of several cases with reduced increase of CD4-cells upon start of antiretroviral treatment (ART) prompted us to investigate the topic using the ClinSurv cohort.

Methods: 17 German HIV treatment centers contribute to ClinSurv, a multicentre observational cohort under the auspices of the Robert Koch Institute. We retrospectively selected all antiretroviral-naïve patients with toxoplasmic encephalitis (TE) and - as comparator group - with pneumocystosis (PCP) between January 1999 and December 2005.
\end{abstract}

Results: A total of 257 patients were included in the analysis, 61 with TE and 196 with PCP. Demographic baseline data showed differences with regard to gender, transmission group, and baseline $\mathrm{CD}^{+}$counts (60.9 vs. $44.7 / \mu 1, p=0.022)$. After ART-initiation the increase in $\mathrm{CD}^{+}$lymphocytes was lower in the TEversus the PCP-group in the first, second and fourth three-month-period (74.4 vs. $120.3 / \mu \mathrm{l}, \mathrm{p}=0.006$; 96.6 vs. $136.2 / \mu \mathrm{l}, \mathrm{p}=0.021 ; 156.5$ vs. $211.5 / \mu \mathrm{l}, \mathrm{p}=0.013)$. Viral load (VL) was higher in the PCP-group at baseline $\left(4.46 \log _{10} \mathrm{cop} / \mathrm{ml}\right.$ vs. $5.00 \log _{10} \mathrm{cop} / \mathrm{ml}, \mathrm{p}=$ 0.008), while virological success of ART was equal. Conclusions: Our data show for the first time that the average $\mathrm{CD}^{+}{ }^{+} \mathrm{T}$-cell increase of patients with toxoplasmosis is impaired compared to PCP-patients. Most clinicians would not be prepared to discontinue follow-up TE-therapy unless CD4+ counts of $200 / \mu$ are reached. Explanation for our finding might be the myelosuppressive side effect of pyrimethamine, possible interactions of toxoplasmosis therapy with ART, or an unknown direct biological influence of toxoplasmosis on immune restoration.

Key words: Antiretroviral therapy, highly active; $\mathrm{CD}^{+}$ lymphocyte count; HIV; pneumocystis jiroveci pneumonia; toxoplasmic encephalitis

List of Abbreviations: AIDS = Acquired immune deficiency syndrome, ART = Antiretroviral therapy, $\mathrm{HPC}=$ Patients from countries of high HIV-prevalence, M1, M2... = Month 1, month 2.., MSM = Men who have sex with men, OI = Opportunistic infection, PCP = Pneumocystis pneumonia, Q1, Q2... $=$ Quarter 1, quarter 2..., TE = Toxoplasmic encephalitis, VL $=$ Viral load

\section{INTRODUCTION}

Although the development of efficient antiretroviral treatment options has changed the face of the AIDS epidemic in industrialized countries, opportunistic infections like toxoplasmic encephalitis or pneumocystis pneumonia still present a considerable challenge [1-3]. Especially in patients in a late stage of HIV infection, symptoms of AIDS-defining diseases are a common reason for first hospital admission [4-6]. While detailed treatment guidelines exist for most opportunistic infections, the situation regarding toxoplasmosis is more difficult. Especially the question when to initiate antiretroviral treatment in order to avoid immune reconstitution problems, and when to stop the anti-infective therapy remains open in most guideline reviews [7]. One reason for this may be the significantly lower incidence of toxoplasmic encephalitis in the USA compared with European countries [8-10]. Only scarce data about immunological and virological response to highly active antiretroviral therapy initiated in patients treated for toxoplasmosis is to be found in the literature. Observation of such patients in the clinical setting led to the hypothesis of an impaired CD4+ lymphocyte response to ART.

\section{Methods}

To further investigate this hypothesis we used the database of the "ClinSurv HIV" cohort, a multicentre cohort of 17 German HIV clinics led by the Robert Koch Institute in Berlin. The data reported biannually includes clinical and laboratory parameters, patient demographics, transmission route, date of HIV-diagnosis, clinical stage, AIDS related or AIDS defining diseases, antiretroviral treatment and date of death. We retrospectively searched this database for patients who matched the following criteria: Diagnosis of either toxoplasmic encephalitis or pneumocystis pneumonia in the period between January 1999 and December 2005; ART-naïve; initiation of ART in the 2 months following the OI-diagnosis; sufficient documentation of ART-regimen, viral load and $\mathrm{CD}^{+}{ }^{+}$T-cell count (defined by at least three measurements of each laboratory parameter) in the 12 months following ART-initiation; and a CD4+ lymphocyte count below 200/ $\mu$ at baseline. PCP-cases were chosen as a control group 
due to the hypothesis that this group would provide a sufficient number of patients with a comparably impaired immunologic status. Diagnosis of PCP was established by detection of Pneumocystis jiroveci in the broncho-alveolar lavage. Toxoplasmosis was diagnosed by brain imaging, clinical signs, serology and response to anti-Toxoplasma treatment according to the European AIDS case definition [11] and the CDC "1993 Revised Classification System for HIV Infection and Expanded Surveillance Case Definition for AIDS Among Adolescents and Adults" [12].

The two groups were compared regarding their demographic characteristics, baseline viral load and $\mathrm{CD}^{+} / \mathrm{CD}^{+}+\mathrm{T}$-cell count, as well as virological and immunological response in the first 12 months of ART. Every laboratory test performed in this period was assigned to a one-month-window (M1, M2, M3 $\ldots$ and M12) and in a second step, because of the high amount of missing values, to a three-month-window (quarter Q1, Q2, Q3 and Q4) starting from the date of ART-initiation. For windows containing more than one value an arithmetic mean was calculated. If a window contained viral-load values both below detection limit $(<50$ copies $/ \mathrm{ml})$ and above, only the latter were taken into account. Virological response to ART was compared using cross tables and Chi-squared test for percentage of patients with a VL below the limit of detection after 6 and 12 months of ART and performing Kaplan-Meier survival analysis for time to vi- ral load suppression (defined by two consecutive VLmeasurements $<50 \mathrm{cop} / \mathrm{ml})$. Mean values of absolute $\mathrm{CD}^{+}$lymphocyte count, total lymphocytes and deltaCD4 (increase in CD4+ ${ }^{+}$-cells in comparison to baseline) were compared by T-test. In order to look at a more clinically relevant parameter for a positive antiretroviral treatment outcome, immunological response was defined as reaching a $\mathrm{CD}^{+}$lymphocyte count of $200 / \mu \mathrm{l}$ or higher and compared performing a Kaplan-Meier analysis regarding time to this event. A uni- and multivariate analysis of factors with possible influence on the probability of immunological response in Q2 was performed. Therefore the odds ratios as estimators of the relative risk to reach immunological response were calculated using cross tables (univariate) and logistic regression analysis (multivariate). The multivariate analysis adjusted for age and sex and included all factors with a $\mathrm{p}<0.2$ in the univariate analysis.

\section{RESULTS}

\section{BASELINE CHARACTERISTICS}

A total of 257 patients within the CLINSURV data set of 17,521 patients enrolled in the cohort at time of data collection met the selection criteria: 61 with diagnosis of toxoplasmosis, and 196 with diagnosis of pneumocystis pneumonia (see Table 1 for details). No

Table 1. Overview of baseline characteristics in the compared groups; $\mathrm{SD}=$ standard deviation, $\mathrm{MSM}=$ men who have sex with men, $\mathrm{HPC}=$ patients from countries of high HIV prevalence, ART $=$ antiretroviral therapy, $\mathrm{IQR}=$ interquartile range, $\mathrm{NNRTI}=$ antiretroviral regimen based on NNRTI, PI $=$ antiretroviral regimen based on PI, NRTI or NNRTI $/$ PI $=$ antiretroviral regimen either consisting of NRTI only or including NNRTI and PI; p-values calculated by T-test, Chi-squared or Mann-Whitney-U test.

\begin{tabular}{|c|c|c|c|c|c|}
\hline & & & & 96) & \\
\hline & $\mathrm{n} /$ mean & $\% / \mathrm{SD}$ & $\mathrm{n} /$ mean & $\% / \mathrm{SD}$ & $\mathrm{p}$ \\
\hline Age & 40.8 & 8.4 & 41.0 & 10.5 & 0.876 \\
\hline Female & 16 & $26.2 \%$ & 28 & $14.3 \%$ & 0.031 \\
\hline Migrant & 13 & $21.3 \%$ & 41 & $20.9 \%$ & 0.948 \\
\hline $\begin{array}{l}\text { Transmission risk } \\
\text { MSM }\end{array}$ & 25 & $41.0 \%$ & 114 & $58.2 \%$ & 0.013 \\
\hline $\mathrm{HPC}$ & 11 & $18.0 \%$ & 14 & $7.1 \%$ & \\
\hline Other & 25 & $41.0 \%$ & 68 & $34.7 \%$ & \\
\hline ART initiation date & $\begin{array}{c}\text { Apr } 02 \\
\text { (median) }\end{array}$ & $\begin{array}{l}34 \text { months } \\
\text { (IQR) }\end{array}$ & $\begin{array}{c}\text { Sep } 01 \\
\text { (median) }\end{array}$ & $\begin{array}{l}35 \text { months } \\
\text { (IQR) }\end{array}$ & 0.631 \\
\hline Firstline-ART & & & & & \\
\hline NNRTI & 11 & $18.0 \%$ & 34 & $17.3 \%$ & 0.069 \\
\hline PI & 45 & $73.8 \%$ & 119 & $60.7 \%$ & \\
\hline NNRTI/PI & 3 & $4.9 \%$ & 37 & $18.9 \%$ & \\
\hline NRTI & 2 & $3.3 \%$ & 6 & $3.1 \%$ & \\
\hline Baseline values & & & & & \\
\hline $\mathrm{CD} 4 / \mu \mathrm{l}$ & 60.9 & 44.2 & 44.7 & 42.1 & 0.022 \\
\hline $\mathrm{CD} 8 / \mu \mathrm{l}$ & 637.2 & 301.2 & 726.4 & 492.3 & 0.222 \\
\hline$\% \mathrm{CD} 4$ & 6.8 & 4.3 & 4.8 & 3.9 & 0.013 \\
\hline$\% \mathrm{CD} 8$ & 67.0 & 13.3 & 65.2 & 15.9 & 0.565 \\
\hline VL $\left(\log _{10} \mathrm{Kop} / \mathrm{ml}\right)$ & 4.46 & 1.20 & 5.00 & 1.02 & 0.008 \\
\hline Lymphocytes/ $\mu \mathrm{l}$ & 981.8 & 544.1 & $1,056.0$ & 887.39 & 0.635 \\
\hline
\end{tabular}


significant differences were shown for age, proportion of patients with migration background, date of ARTinitiation, baseline CD8-cell-count and total lymphocyte count.

The percentage of female patients in the TE-group was significantly higher than in the PCP-group. The distribution of patients over the different HIV transmission risk categories also diverged significantly, especially with regards to the proportion of men who have sex with men (MSM) and patients from countries of high HIV-prevalence (HPC). In both groups, ARTregimens based on a boosted protease inhibitor (PI) were chosen as first line antiretroviral treatment in more than $60 \%$ of the cases, followed in frequency by non-nucleoside reverse transcriptase inhibitor (NNRTI) based regimens. An unusually high amount of PCP-patients was initially treated with a combination including both PI and NNRTI. Almost $50 \%$ of the TE-patients started antiretroviral treatment within a month after OI-diagnosis, while in over $60 \%$ of the PCP-group ART-initiation was delayed until the $1^{\text {st }}$ month after diagnosis.

The TE-patients in this analysis showed a higher absolute and relative CD4-cell count at baseline compared to the PCP-group. At the same time the baseline viral load was significantly lower in the TE-group.

\section{Virological And ImmunOlogical Response}

Virologic response to ART was comparable in both groups; not only regarding the percentage of patients with viral load below detection limit after $6(68.4 \% \mathrm{vs}$. $77.5 \%, \mathrm{p}=0.279)$ and 12 months $(87.5 \%$ vs. $77.5 \%, \mathrm{p}$ $=0.369)$, but also comparing both groups in regards to "time to the second consecutive HIV-PCR with a result of $<50 \mathrm{cop} / \mathrm{ml}$ ". The Kaplan-Meier analysis showed a median time to this event of 8 months in both groups ( $p=0.919$, log-rank test).

Absolute $\mathrm{CD}^{+}$lymphocyte count, delta-CD4, and total lymphocyte count were numerically lower in the TE-group in all 4 quarters. In Q1, TE-patients showed a significantly lower absolute $\mathrm{CD}^{+}$lymphocyte count $(129.1 / \mu \mathrm{l}$ vs. $163.6 / \mu \mathrm{l}, \mathrm{p}=0.043)$ as well as a reduced $\mathrm{CD}^{+}{ }^{+}$T-cell increase $(+74.4 / \mu \mathrm{l}$ vs. $+120.3 / \mu \mathrm{l}$, $\mathrm{p}=0.006)$ and less total lymphocytes $(1,205.3 / \mu \mathrm{l}$ vs. $1,669.4 / \mu 1, \mathrm{p}<0.001)$. In the $2^{\text {nd }}$ and $4^{\text {th }}$ quarter, only delta-CD4 and total lymphocyte count remained significantly impaired in the TE-group (see Fig. 1).

The percentage of TE-patients reaching an average $\mathrm{CD}^{+}$lymphocyte count of at least $200 / \mu \mathrm{l}$ was well below that of PCP-patients in Q1 and Q2, while in the second half of the observation period this difference seems to disappear (see Fig. 2). A Kaplan-Meier analysis comparing time to the event "first absolute $\mathrm{CD}_{4}^{+}$ lymphocyte count $\geq 200 / \mu \mathrm{l}$ " showed a non-significant advantage for the PCP-group with a median time to event of 8 months (vs. 10 months in the TE-group, p $=0.269$, log-rank test).

To identify other factors possibly related with reduced immunological response, uni- and multivariate analyses were performed. Besides TE (vs. PCP) they included the factors age, gender, MSM, HPC, PI- and NNRTI-based first-line ART-regimen, date of ART-

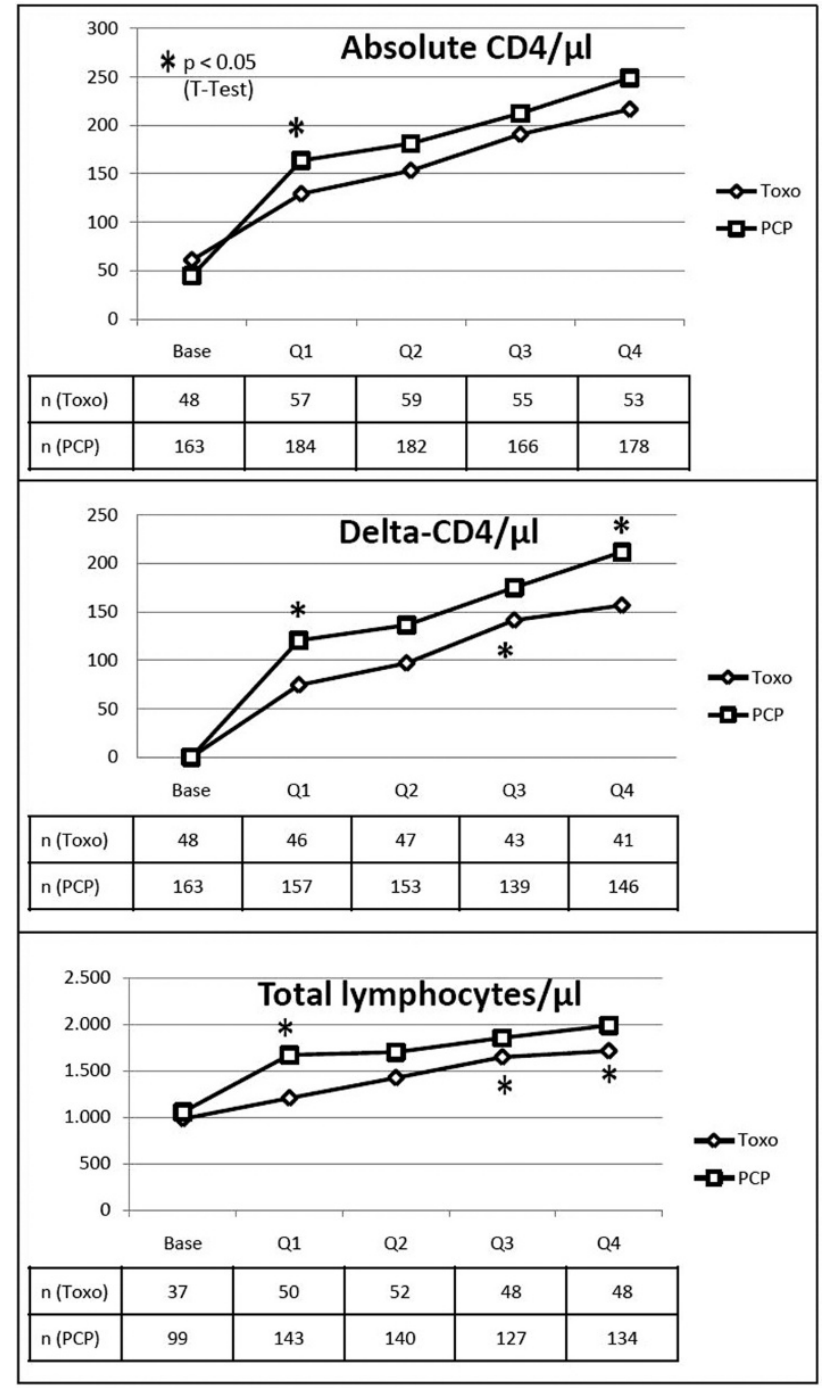

Fig. 1. Course of mean absolute CD4-cell count, delta-CD4 and total lymphocytes through the four quarters of the observation period; TE patients show reduced values in all three parameters. $*=\mathrm{p}<0.05$

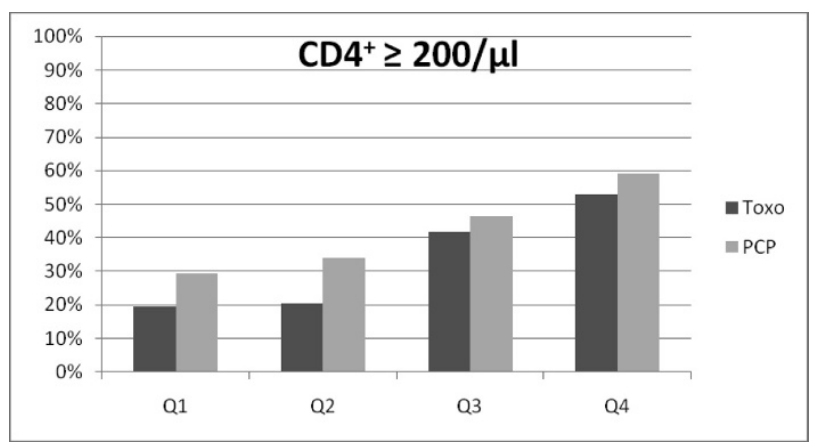

Fig. 2. Lower proportion of TE patients with immunologic response, defined as average CD4-cell count $\geq 200 / \mu$, in Q1 $(19.3 \%$ vs. $29.3 \%, \mathrm{p}=0.135)$ and $\mathrm{Q} 2(20.3 \%$ vs. $34.1 \%, \mathrm{p}=$ 0.047 , calculated by Chi-squared test).

initiation, baseline viral load and baseline CD4+ ${ }^{+}$T-cell count. Of all these factors only diagnosis of toxoplasmosis and a baseline $\mathrm{CD}^{+}{ }^{+}$lymphocyte count below 50 cells $/ \mu$ l were associated with a significantly lower probability of reaching the defined $\mathrm{CD}^{+}$goal of 
Table 2. Uni- and multivariate analysis showing significant influence on immunologic response, here defined as average CD4cell count $\geq 200 / \mu \mathrm{l}$ in Q2, only for the factors TE and baseline CD $4<50 / \mu \mathrm{l}$.

\begin{tabular}{|c|c|c|c|c|}
\hline \multirow[b]{2}{*}{ Factor } & \multicolumn{2}{|c|}{ Univariate analysis } & \multicolumn{2}{|c|}{ Multivariate Analysis } \\
\hline & odds ratio & $\mathrm{p}$ & odds ratio & $\mathrm{p}$ \\
\hline TE (vs. PCP) & 0.576 & 0.047 & 0.403 & 0.033 \\
\hline Age $\geq 40($ vs. $<40)$ & 1.308 & 0.067 & 1.582 & 0.171 \\
\hline Female gender (vs. male) & 0.827 & 0.555 & 0.931 & 0.872 \\
\hline MSM (vs. other transmission risk) & 1.141 & 0.29 & & \\
\hline HPC (vs. other transmission risk) & 0.797 & 0.614 & & \\
\hline PI-based first line ART (vs. other) & 0.961 & 0.708 & & \\
\hline NNRTI-based first line ART (vs. other) & 0.978 & 0.941 & & \\
\hline ART-initiation before $11 / 01$ (vs. $11 / 01$ and later) & 0.821 & 0.176 & 0.926 & 0.816 \\
\hline Baseline VL $>5 \log _{10}\left(\right.$ vs. $\left.\leq 5 \log _{10}\right)$ & 1.148 & 0.318 & & \\
\hline Baseline CD4 <50/ $\mu$ l (vs. $\geq 50 / \mu \mathrm{l})$ & 0.603 & $<0.001$ & 0.281 & $<0.001$ \\
\hline
\end{tabular}

$200 / \mu$ in Q2. This effect remained also significant in the multivariate analysis adjusting for age, sex and date of ART-initiation (as the only factor with a $\mathrm{p}<0.2$ in the univariate analysis) (see Table 2).

\section{Discussion}

Our analysis shows that immunological response to ART initiated in patients with diagnosis of toxoplasmosis is impaired when compared to patients with PCP. This difference comprises lower absolute CD4+ lymphocyte counts and delta-CD4, as well as a significantly reduced probability of reaching $\geq 200 \mathrm{CD}^{+}{ }^{+} \mathrm{T}$ cells $/ \mu \mathrm{l}$ in the second quarter of the observed first year under ART. This negative effect gets weaker (and disappears) in the second half-year period following the start of ART. The observed lower $\mathrm{CD}^{+}$lymphocyte count seems to derive mainly from a reduced total lymphocyte count in the TE-group. A plausible explanation could be the known myelosuppressive side effects of anti-infective agents used as recommended standard treatment for toxoplasmosis (pyrimethamine) [13-15]. The substitution of folic acid routinely provided during TE-therapy with pyrimethamine only partially prevents this side effect. The standard PCP treatment with cotrimoxazole can impair the bone marrow as well, but it has a smaller myelosuppressive potency than pyrimethamine, furthermore the suggested duration of PCP-therapy is shorter than for TE $[7,16]$.

If this conclusion will prove correct in further studies, guidelines suggesting a solely CD4-cell guided duration of maintenance therapy would have to be reviewed, in order to avoid a sort of "vicious circle": Low $\mathrm{CD}^{+}$T-cell count leads to ongoing pyrimethamine-treatment leads to low $\mathrm{CD}^{+}{ }^{+}$T-cell count etc. D'Egidio et al. have already showed that with sustained viral load suppression the termination of secondary PCP-prophylaxis should be possible also for patients with a CD4-cell count below 200/ $\mu$ l [17]. To evaluate a similar concept for toxoplasmic encephalitis further research is necessary. Another consequence should be an intensified search for alternative anti-toxoplasmosis drugs with less hematotoxic side effects [18-21], as well as strategies to directly en- hance $\mathrm{CD}^{+}$lymphocyte increase, always considering the possibility of multiple drug interactions in this setting of extensive treatment $[22,23]$.

A less probable reason for the observed difference would be a direct interaction of the opportunistic infection with the immune system, for example by suppressing myelopoiesis, as it has been shown for generalized infection with Mycobacterium avium complex (MAC) [25-27]. A similar myelosuppressive effect caused by Toxoplasma has been demonstrated in the acute infection in mice. Finally, the use of corticosteroids in initial PCP-therapy could be proposed as a reason for higher CD4-cell counts. There have been reports from small HIV-patient populations showing an increase in CD4-cells when patients were treated with prednisolone [29, 30]. As exact data about steroid use in both groups is lacking, we did not include this factor in our analysis.

\section{LIMITATIONS}

Due to the retrospective nature of the ClinSurv cohort, the authors could not confirm each diagnosis of TE / PCP by assessing the diagnostic procedures that were applied. Diagnosis was performed by the clinicians at the treatment centers who were asked to report only cases of confirmed TE / PCP diagnosis, established according to the above mentioned guidelines. Especially for TE a bias due to incorrectly diagnosed OI cannot be ruled out.

As mentioned above the compared groups significantly diverged along the following baseline characteristics: gender, HIV-transmission group, first line ARTregimen, time from OI diagnosis to ART-initiation, baseline $\mathrm{CD}^{+}{ }^{+}$lymphocyte count and viral load. These differences are difficult to explain and may mainly result from the low number of cases or from a bias due to incorrectly diagnosed OI. A reason for the higher percentage of patients from HPC in the TE-group could be a higher seroprevalence of Toxoplasma gondii in hot and humid regions and countries with lower hygiene standards [9]. An explanation for the uncommon choice of first-line ART in the PCP group (18.9\% PI/NNRTI containing regimen) may be a higher baseline viral load and lower baseline $\mathrm{CD}^{+}$lymphocyte 
count in that treatment-group $\left(\log _{10} \mathrm{VL} 5.21\right.$ vs. $4.82 \mathrm{cop} / \mathrm{ml}, \mathrm{p}=0.007, \mathrm{CD}^{+} 36.9$ vs. $50.8 / \mu \mathrm{l}, \mathrm{p}=$ 0.075 , compared to all patients with different first-line ART) as rationale for a more intense ART-regimen. The different time to ART-initiation may have its cause in the treatment guidelines: Waiting until the initial anti-infective treatment shows clinical response is suggested for several OI including PCP, while for toxoplasmosis clear indications for the ideal time to start with ART are missing in the guidelines [7, 31].

In a sensitivity analysis adjusting for all these factors, the above mentioned influence of a low $\mathrm{CD}^{+}{ }^{+} \mathrm{T}$ cell count $(<50 / \mu \mathrm{l})$ on the probability of reaching $\geq 200 \mathrm{CD}^{+} / \mu \mathrm{l}$ proved highly significant, "TE" only marginally missed significance-level in this analysis ( $p$ $=0.059)$. Therefore, the risk of a bias caused by these differences should be negligible.

Regarding the differences in baseline VL and CD4cell count, there is some evidence that a lower viral load and/or a lower CD4-cell count before starting ART is associated with a higher risk of discordant immunologic and virologic response to treatment [24]. This leaves us with the dilemma that, on the one hand, the lower baseline viral load in the TE group could have led to the impaired CD4-cell increase in this group, while on the other hand the higher baseline CD4-cell count in the TE patients should have had the opposite effect.

Data collection for the ClinSurv cohort consists of a preselected catalogue of parameters. This catalogue does not necessarily take into account all information which could have been of interest for some of the questions this retrospective analysis brought up. Not recorded were data like the kind and duration of antiinfective treatment or ART adherence. Further, as ClinSurv is an observational cohort, data collection is not connected to predefined follow-up time points. Therefore laboratory parameters like CD4+ lymphocyte count or viral load are measured in very divergent intervals. The only inclusion criteria regarding quantity of laboratory data for this analysis were a minimum of three CD4 ${ }^{+}$T-cell count and viral load measurements performed during the observation period. Consequently some of the patients contribute information to the analysis only for part of the year observed. Furthermore calculating averages for quarters with more than one lab value could have led to a bias by overrating single values. Excluding patients with missing values for one or more of the four quarters would have led to a substantial loss of information. On the other hand the minimum quantity of measurements could have led to a selection bias towards patients with favorable courses of their diseases, taking into account that none of the selected patients died during the oneyear follow-up period.

\section{CONCLUSION}

Toxoplasma encephalitis seems to be associated with an impaired immunological response to ART compared to patients with pneumocystis pneumonia. This regards mainly the first months after ART-initiation and may be caused by the myelosuppressive side effects of anti-toxoplasmosis drugs. Further investigations about this effect including direct biological influ- ence of toxoplasmosis on immune restoration and drug interactions between ART and anti-infective treatment are of great interest. The search for new drugs against Toxoplasma gondii should be intensified as well. With respect to clinical management, other hematotoxic drugs should be avoided in this setting. Guidelines regarding time of initiation and duration of anti-toxoplasmosis treatment should be critically reviewed, especially to avoid the "vicious circle" of decisions guided solely by CD4-cell count.

\section{Acknowledgements:}

\section{Participating centers:}

Berlin: PD Dr. K. Arastéh, S. Kowol: Auguste-ViktoriaKlinikum; Dr. F. Bergmann, M. Warncke: Charité Campus Virchow; Bochum: Prof. Dr. N. Brockmeyer, N. Mühlbächer: Ruhr Universität Bochum; Bonn: Prof. Dr. J. Rockstroh, Dr. J. Wasmuth: Universitätsklinikum Bonn; Düsseldorf: Dr. M. Oette, C. Blondin: Universitätsklinik Düsseldorf; Essen: Dr. S. Esser, P. Schenk-Westkamp: Universitätsklinik Essen; Hamburg: Prof. Dr. A. Plettenberg, T. Lorenzen, I. Walther: ifi; Drs. A. Adam/ L. Weitner/ K. Schewe/ H. Goey, Drs. S. Fenske/ T. Buhk/ HJ. Stellbrink, Dr. H. Gellermann: IPM Studycenter; PD. Dr. J. van Lunzen, D. Elshani; Universitätsklinikum Eppendorf; Hannover: Prof. Dr. M. Stoll, S. Gerschmann: Medizinische Hochschule Hannover; Kiel: Prof. Dr. H. Horst: Universitätsklinik Kiel; Köln: Prof. Dr. G. Fätkenheuer, T. Kümmerle, D. Gillor: Universitätsklinik Köln; München: Prof. Dr. Bogner, B. Sonntag: Universitätsklinikum München; Regensburg: Prof. Dr. B. Salzberger: Universitätsklinik Regensburg; Rostock: Dr. C. Fritzsche; Universitätsklinik Rostock

Cohort manager: A. Kuehne, Robert Koch Institute, Berlin, Germany

The ClinSurv study is primarily funded by the Robert KochInstitute and was partly funded by a grant from the German Federal Ministry of Education and Research (2003-2005), and the German Federal Ministry of Health (1999).

\section{REFERENCES}

1. Abgrall S, Rabaud C, Costagliola D. Incidence and risk factors for toxoplasmic encephalitis in human immunodeficiency virus-infected patients before and during the highly active antiretroviral therapy era. Clin Infect Dis 2001 Nov 15;33(10):1747-55.

2. San-Andres FJ, Rubio R, Castilla J, Pulido F, Palao G, de $\mathrm{P}$, I, et al. Incidence of acquired immunodeficiency syndrome-associated opportunistic diseases and the effect of treatment on a cohort of 1115 patients infected with human immunodeficiency virus, 1989-1997. Clin Infect Dis 2003 May 1;36(9):1177-85.

3. Brodt HR, Kamps BS, Gute P, Knupp B, Staszewski S, Helm EB. Changing incidence of AIDS-defining illnesses in the era of antiretroviral combination therapy. AIDS 1997 Nov 15;11(14):1731-8.

4. Battegay M, Fluckiger U, Hirschel B, Furrer H. Late presentation of HIV-infected individuals. Antivir Ther 2007;12(6):841-51.

5. Keruly JC, Moore RD. Immune status at presentation to care did not improve among antiretroviral-naive persons from 1990 to 2006. Clin Infect Dis 2007 Nov 15;45(10): 1369-74.

6. Pulvirenti J, Herrera P, Venkataraman P, Ahmed N. Pneumocystis carinii pneumonia in HIV-infected patients in the HAART era. AIDS Patient Care STDS 2003 Jun; $17(6): 261-5$. 
7. Benson CA, Kaplan JE, Masur H, Pau A, Holmes KK. Treating opportunistic infections among HIV-infected adults and adolescents: recommendations from CDC, the National Institutes of Health, and the Infectious Diseases Society of America. Webpage 2004Available from: URL: http://aidsinfo.nih.gov/contentfiles/TreatmentofOI_AA.pdf

8. Clumeck N. Some aspects of the epidemiology of toxoplasmosis and pneumocystosis in AIDS in Europe. Eur J Clin Microbiol Infect Dis 1991 Mar;10(3):177-8.

9. Montoya JG, Liesenfeld O. Toxoplasmosis. Lancet 2004 Jun 12;363(9425):1965-76.

10. Israelski DM, Chmiel JS, Poggensee L, Phair JP, Remington JS. Prevalence of Toxoplasma infection in a cohort of homosexual men at risk of AIDS and toxoplasmic encephalitis. J Acquir Immune Defic Syndr 1993 Apr;6(4): 414-8.

11. Ancelle-Park R. Expanded European AIDS case definition. Lancet 1993 Feb 13;341(8842):441.

12. 1993 revised classification system for HIV infection and expanded surveillance case definition for AIDS among adolescents and adults. MMWR Recomm Rep 1992 Dec 18;41(RR-17):1-19.

13. Nath A, Sinai AP. Cerebral Toxoplasmosis. Curr Treat Options Neurol 2003 Jan;5(1):3-12.

14. Masters PA, O'Bryan TA, Zurlo J, Miller DQ, Joshi N. Trimethoprim-sulfamethoxazole revisited. Arch Intern Med 2003 Feb 24;163(4):402-10.

15. Freund YR, Dabbs J, Creek MR, Phillips SJ, Tyson CA, MacGregor JT. Synergistic bone marrow toxicity of pyrimethamine and zidovudine in murine in vivo and in vitro models: mechanism of toxicity. Toxicol Appl Pharmacol 2002 May 15;181(1):16-26.

16. Kirk O, Reiss P, Uberti-Foppa C, Bickel M, Gerstoft J, Pradier C, et al. Safe interruption of maintenance therapy against previous infection with four common HIV-associated opportunistic pathogens during potent antiretroviral therapy. Ann Intern Med 2002 Aug:\%\%20;137(4):239-50.

17. D'Egidio GE, Kravcik S, Cooper CL, Cameron DW, Fergusson DA, Angel JB. Pneumocystis jiroveci pneumonia prophylaxis is not required with a $\mathrm{CD} 4{ }^{+} \mathrm{T}$-cell count $<$ 200 cells/microl when viral replication is suppressed. AIDS 2007 Aug;\%20;21(13):1711-5.

18. Linares GG, Gismondi S, Codesido NO, Moreno SN, Docampo R, Rodriguez JB. Fluorine-containing aryloxyethyl thiocyanate derivatives are potent inhibitors of Trypanosoma cruzi and Toxoplasma gondii proliferation. Bioorg Med Chem Lett 2007 Sep 15;17(18):5068-71.

19. el Kouni MH. Adenosine metabolism in Toxoplasma gondii: potential targets for chemotherapy. Curr Pharm Des 2007;13(6):581-97.

20. Shubar HM, Mayer JP, Hopfenmuller W, Liesenfeld O. A new combined flow-cytometry-based assay reveals excellent activity against Toxoplasma gondii and low toxicity of new bisphosphonates in vitro and in vivo. J Antimicrob Chemother $2008 \mathrm{Feb} \%$ 19;

21. Moreno SN, Li ZH. Anti-infectives targeting the isoprenoid pathway of Toxoplasma gondii. Expert Opin Ther Targets 2008 Mar;12(3):253-63.

22. Durier C, Capitant C, Lascaux AS, Goujard C, Oksenhendler E, Poizot-Martin I, et al. Long-term effects of intermittent interleukin-2 therapy in chronic HIV-infected patients (ANRS 048-079 Trials). AIDS 2007 Sep 12;21(14):1887-97.
23. Goldberg GL, Zakrzewski JL, Perales MA, van den Brink MR. Clinical strategies to enhance $\mathrm{T}$ cell reconstitution. Semin Immunol 2007 Oct 25;.

24. Moore DM, Hogg RS, Yip B, Wood E, Tyndall M, Braitstein $\mathrm{P}$, et al. Discordant immunologic and virologic responses to highly active antiretroviral therapy are associated with increased mortality and poor adherence to therapy. J Acquir Immune Defic Syndr 2005 Nov 1;40(3):28893.

25. Hafner R, Inderlied CB, Peterson DM, Wright DJ, Standiford HC, Drusano G, et al. Correlation of quantitative bone marrow and blood cultures in AIDS patients with disseminated Mycobacterium avium complex infection. J Infect Dis 1999 Aug;180(2):438-47.

26. Lazaro E, Coureau G, Guedj J, Blanco P, Pellegrin I, Commenges $\mathrm{D}$, et al. Change in T-lymphocyte count after initiation of highly active antiretroviral therapy in HIV-infected patients with history of Mycobacterium avium complex infection. Antivir Ther 2006;11(3):343-50.

27. Kremer L, Estaquier J, Wolowczuk I, Biet F, Ameisen JC, Locht C. Ineffective cellular immune response associated with T-cell apoptosis in susceptible Mycobacterium bovis BCG-infected mice. Infect Immun 2000 Jul;68(7):426473.

28. Petakov M, Stojanovic N, Jovcic G, Bugarski D, Todorovic V, Djurkovic-Djakovic O. Hematopoiesis during acute Toxoplasma gondii infection in mice. Haematologia (Budap ) 2002;32(4):439-55.

29. Andrieu JM, Lu W, Levy R. Sustained increases in CD4 cell counts in asymptomatic human immunodeficiency virus type 1 -seropositive patients treated with prednisolone for 1 year. J Infect Dis 1995 Mar;171(3):523-30.

30. Ulmer A, Muller M, Bertisch-Mollenhoff B, Frietsch B. Low dose prednisolone reduces $\mathrm{CD}^{+} \mathrm{T}$ cell loss in therapy-naive HIV-patients without antiretroviral therapy. Eur J Med Res 2005 Mar 29;10(3):105-9.

31. Manzardo C, Zaccarelli M, Aguero F, Antinori A, Miro JM. Optimal timing and best antiretroviral regimen in treatment-naive HIV-infected individuals with advanced disease. J Acquir Immune Defic Syndr 2007 Sep;46 Suppl 1:S9-18.:S9-18.

Received: November 7, 2008 / Accepted: March 28, 2009

\author{
Address for correspondence: \\ U. Kastenbauer \\ Department of Infectious Diseases \\ University Hospital Munich \\ Downtown Campus \\ Medizinische Poliklinik \\ Pettenkoferstrasse 8a \\ 80336 München \\ Germany \\ Email: ulrich.kastenbauer@med.uni-muenchen.de
}

